\title{
Five Phases Music Therapy (FPMT) in Chinese Medicine: Fundamentals and Application
}

\author{
Hui Zhang ${ }^{12}$, Han Lai ${ }^{1 *}$ \\ 1. Chengdu University of TCM, Chengdu, China \\ 2. AkupunkturAkademiet, Aabyhoej, Denmark
}

Corresponding author at Han Lai, Email: laihan1987@163.com, Address: Chengdu University of TCM, Liutai Road 1166, Wenjiang District, Chengdu, China, 611137

\begin{abstract}
Music therapy has served as complementary and alternative medicine for various neurological disorders. Five Phases Music Therapy (FPMT) employs the theory of five phases and five music scales or tones (宫 Gong (do), 商 Shang (ri), 角 Jue (mi), 徵 Zhi (so) and 羽 Yu (la)) to analyze and treat mind-body illness. In Chinese Medicine $(\mathrm{CM})$, the five music scales are used to connect the human body and the universe, interpret personalities and constitution and analyze the influences of climatic changes on health. FPMT has a self-contained theory and routine of practice application. Large amounts of clinical and fundamental reports have been available and clinical benefits have been obtained. However more systemic clinic research esp. evidence-based and random controlled trials must be performed to validate and optimize its routines and biological and neurological mechanism must be further explored. It's reasonable to believe that the effective music therapy will attract more attention from the world outside China with the introduction of FPMT.
\end{abstract}

Keywords: emotion and will; music therapy; five phases, five phases music therapy; psychology

\section{Background}

According to the definition by American Music Therapy Association (AMTA), music therapy employs interventions to accomplish individual goals within a therapeutic relationship by a professional who has completed an approved music therapy program (Kim, 2013). For centuries, music therapy has served as an alternative and complementary approach for various neurological disorders, including stroke, aphasia, visual neglect, Parkinson's disease, dementia, autism, and pain syndrome (Furuya, 2013, Maratos and Gold, 2008). Besides, its effectiveness has been validated by clinic practitioners and researchers worldwide (Xu et al. 2017). Music therapy triggers neuroplasticity, repairs brain damage and regulates the cognitive, emotional and social disorders (Thompson and Schlaug 2015). Music therapy is usually considered as a 
subdivision of psychotherapy, esp. art pyschotherapy, which can be solely or jointly applied to intervene mental disorders (Castillo-Pérez et al., 2010). In Chinese Medicine $(\mathrm{CM})$, psychology and physiology are regarded as an inseparable unity, which means in the etiology, pathogenesis and treatment, psychological and physiological changes are mutually affected (Karchmer 2013). Over 2000 years, CM has been employing many non-physiological approaches in its therapeutic spectrum, e.g. music therapy, linguistic therapy, kinesitherapy, etc. (Scheid, 2013; Zhang, 2010). Additionally, music theories have been widely used in CM not only to analyze and explain an illness condition but also to treat mind-body disorders (Liao et al., 2013).

\section{Five Phases Music Therapy in Chinese Medicine}

Five Phases Music Therapy (FPMT), also called Chinese Medicine Music Therapy (CMMT) employs the theory of five phases and five music scales to analyze and treat mind-body illness. The five music scales i.e. Gong (do), Shang (re), Jue (mi), Zhi (sol), $\mathrm{Yu}$ (la) were firstly defined in Yue Ji (translated as Chapter of Music), which is the $19^{\text {th }}$ chapter in Li Ji or The Book of Rites (collected by Dai Sheng from 51 B.C. to 21 B.C.) and the first systemic theoretic records on music (Sung, 2001). In this chapter, the medical effects of music have already been involved.

The model of five phases i.e. metal, wood, water, fire and earth is one of the most basic philosophic foundations in CM (Flower, et al.,2016). In Yellow Emperor's Inner Classic, a connection of the five phases, five Zang-organs, and human sound with the five scales has been established (Figure 1). It is also explained that Jue, Zhi, Gong, Shang, and Yu are derived from the very primary sound in the beginning of the universe. As recorded in the classic, each organ has its corresponding sound in nature. This relationship is based on the unique holistic understanding in CM that human being and the nature are unified. Music is the bridge of human body and the universe. There are five scales and six temperaments in nature and there are five Zang-organs and six Fu-organs in the human body correspondingly. Pathologically, harmful sound of Gong, Shang, Jue, Zhi and Yu tends to cause spleen, lung, liver, heart, and kidney disorders respectively. 


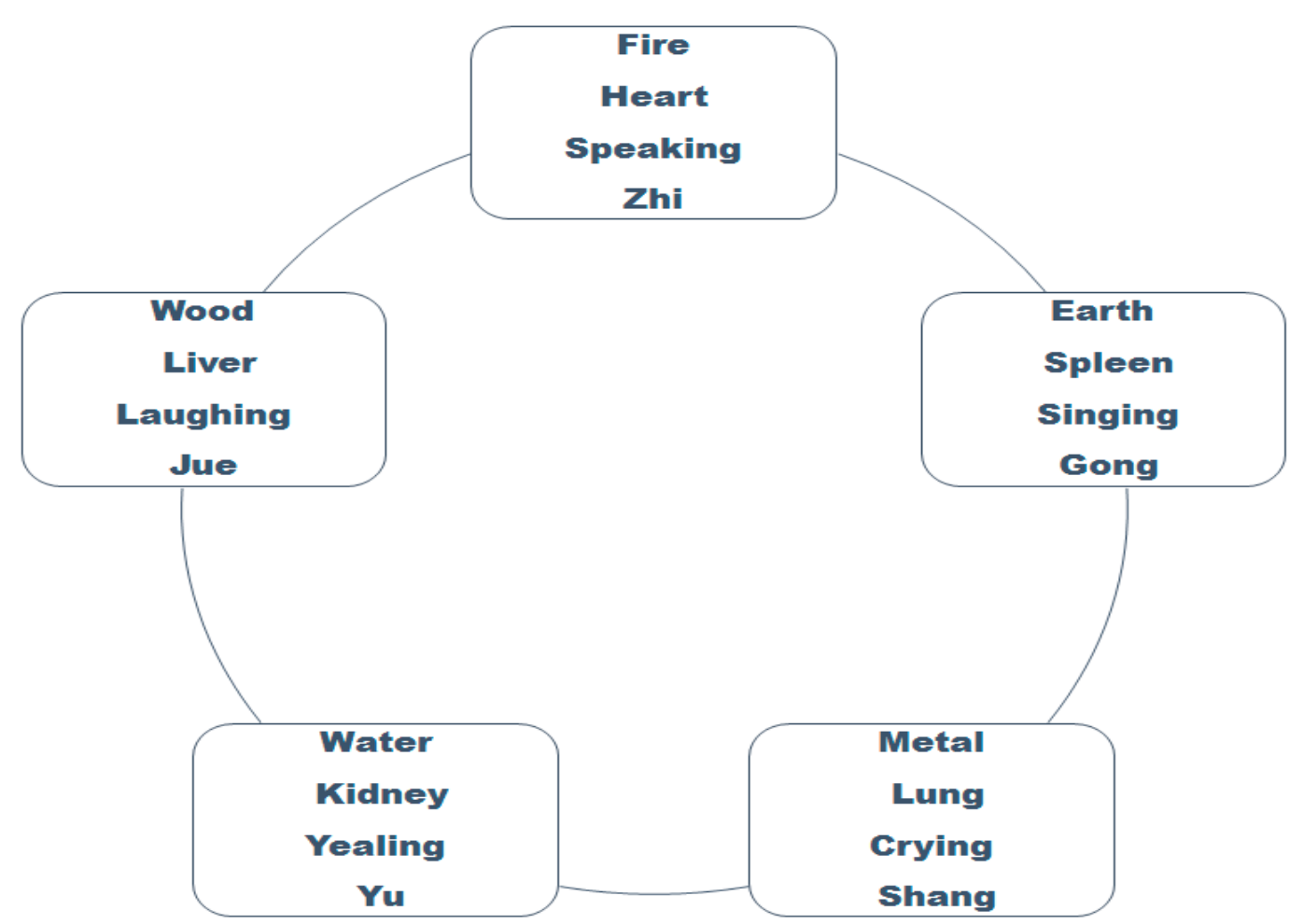

Figure 1. Classification of the five phases, five organs, human sounds, and five scales.

In CM, the model of five music scales is used to interpret the personality and constitution characteristic of individuals (Wang and Zhu, 2009). There are five main types and each type is further divided into five sub-types. Altogether, the 25 types of personality and constitution are defined. In Yun-Qi theory which includes five Yun (wood, fire, earth, metal, water) and six Qi (wind, cold, summer, damp, dryness, fire) to analyze the climatic changes and their relationship to illness on human body. Briefly, Yun-Qi theory can be used to analyze the etiology and predict the onset of a disease (Ma et al., 2013). The five music scales are applied to interpret a climatic feature. For instance, Gong is the sound of Yang-earth and Yin-earth. The Yang Earth corresponds to Tai Gong (greater Gong) and the Yin Earth to Shao Gong (lesser Gong). Tai Gong generates Yin-metal. Thus, the fives sounds are systemically applied to analyze the temporal and spatial variation of the nature and human illnesses (Gong, 2003).

\section{Mechanism of Five Phases Music Therapy}

Music is regarded as a universal feature of human societies, owing to its ability to trigger strong emotions and affect moods (Koelsch, 2014). Music therapy has been widely used in coping mental disorders, such as depression, anxiety, insomnia, schizophrenia, phobia, burnout, etc. The mental disorders belong to the emotion and will diseases (Zhang et al., 2015 and Scheid, 2013). Music with the parameters of pitch, frequency, timbre, and volume affects the emotion, regulates the dynamics of Qi 
movement including ascending, descending, exiting and entering.

In CM, emotions are closely related to organs. Specifically, thinking corresponds to the spleen (Gong), sorrow/worry to the lungs (Shang), anger to the liver (Jue), joy to the heart (Zhi) and fear/fright to the kidneys (Yu) respectively (Cui et al.,2016). The five scales and human emotions are regarded as manifestations of Qi movements and changes. Emotion-will plays a basic role in the onset, progress and prognosis of almost all diseases, not only the psychological and psychiatric disorders. The emotion-will overcoming therapy (EWOT) derived from the philosophical foundation of Chinese medicine, i.e. Yin and Yang, and Five Elements, is believed to be the most effective modality in dealing with mental illness (Zhang et al., 2014).

Shen (spirit) is the reflection of vitality of the human body (Hsu, 2000). Shen governs all psychological and physiological activities. Therefore, priority must be given to heal Shen in all therapeutic approaches in Chinese Medicine, esp. in acupuncture treatment (Moss, 1999). Music is regarded as the best connection with Shen in all cultures (Gaok 2015 and Miller 2013). In CM, the psychological and physiological processes can be translated into Qi, blood, emotion and cognition. Thus, a model is established, showed in figure 2 .

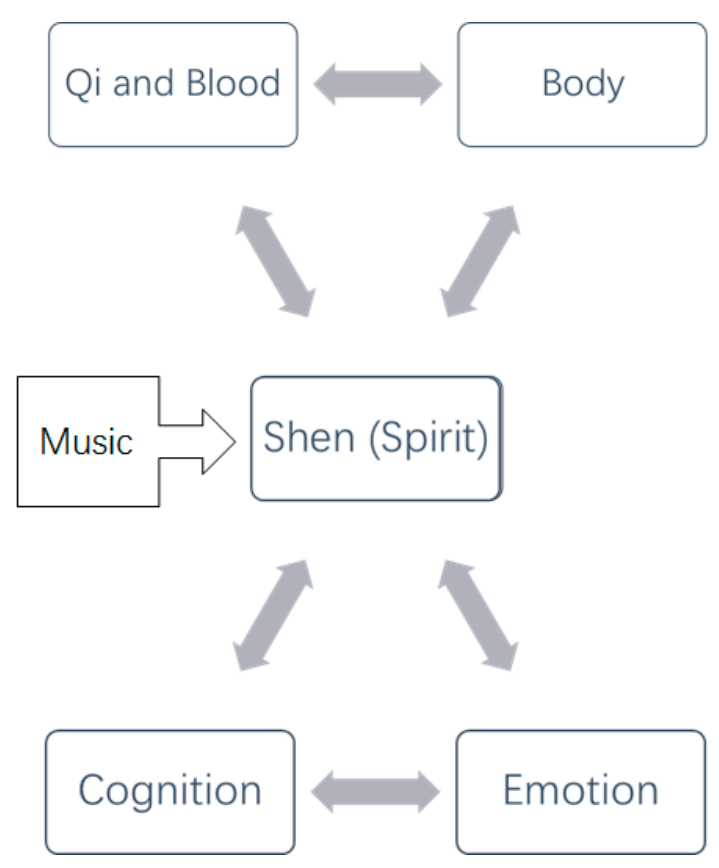

Figure 2. Network model of music therapy in Chinese Medicine.

Therefore, music is called remedy by Zhu Danxi (1281-1358 A.D.), the founder of School of Minister Fire. Interestingly, the two Chinese characters of music and herbal medicine in Chinese language looks very similar (Figure 3) (Cui et al.,2016). For 
example, music could significantly treat the burnout symptoms (Brandes et al., 2009).

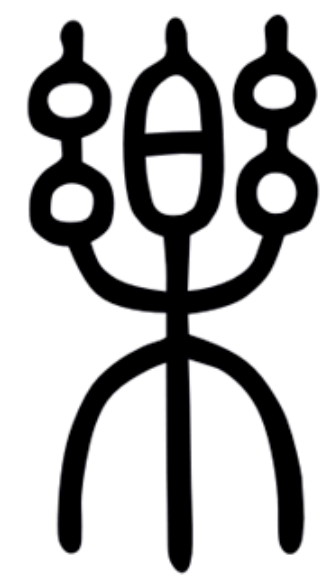

A. Music

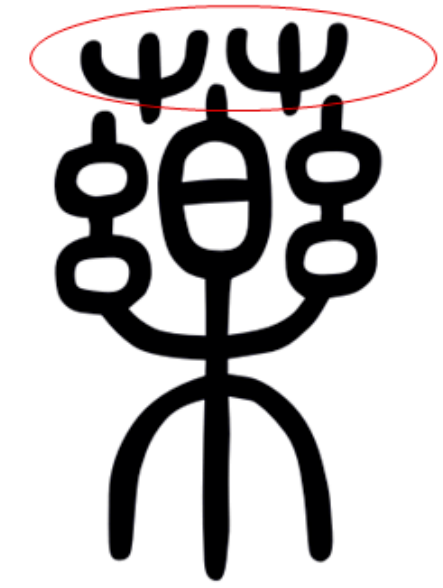

Grass

\section{B.Herbal Medicine}

Figure 3. A: music; B. Herbal medicine. The Chinese character of B has an additional part of "grass" above when compared with A.

\section{Jue (mi) and Anger}

Anger corresponds to the phase of wood and related to the liver. This category of music /song is characterized by being vigorous, melodious, cheerful, and bright. So it's called the music of the spring. When the reaction is hyperactive, anger drives Qi upwards, leading to rebellions of liver Qi, lung Qi, stomach Qi etc. While the anger within normal range activates the free flow of Qi. A psychological disorder stimulated by anger or manifested as anger normally hurts the liver. Music of Jue scale has the function of lifting Qi and soothing liver Qi. Therefore, Qi blockage can be released.

\section{Zhi (so) and Joy}

Joy or happiness corresponds to the phase of fire and related to the heart. This category of music /song is characterized by being warm, cheerful, relaxing, and contagious. So it's called the music of the summer. When the reaction is hyperactive, joy scatters the Shen, leading to distraction, restlessness, palpitation, insomnia, insanity. While joy within normal range facilitates the circulation of blood and inspires spirits. Thus, Music of Zhi invigorates heart Yang, expels cold and regulates Qi and blood.

\section{Gong (do) and Thinking}

Thinking corresponds to the phase of fire and related to the heart. This category of music /song is characterized by being calm, solemn, mellow and heavy. So it's called the music of the late summer. When a person thinks too much or suffers entanglement, Qi is bounded and symptoms of digestive system may appear. The music of Gong 
strengthens the spleen and stomach and improves the ascending and descending of Qi dynamic of the middle Jiao.

\section{Shang (ri) and Sorrow}

Sorrow corresponds to the phase of metal and related to the lungs. This category of music/song is characterized by being resounding, sorrowful, solemn, sonorous and forceful. So, it's called the music of autumn. When the reaction is hyperactive, sorrow consumes Qi. The music of Shang strengthens the astringing and purifying effects of the lungs and regulates water metabolism.

\section{Yu (la) and Fear}

Fear corresponds to the phase of water and related to the kidneys. This category of music/song is characterized by being pure, plaintive, desolate, and gentle. So, it's called the music of winter. When the reaction is hyperactive, fear drives Qi downwards. The music of Yu helps the kidneys to consolidate Qi and essence, improve intelligence and memory and prevent aging.

Based on the framework above, CD series composed by Mr. Shi Feng and played by National Central Musical College Orchestra has been published by Chinese Medical Multimedia Press Co.,Ltd. and widely used in clinic practice (Li et al., 2015, Zhang et al., 2014). The CD series is also recommended by Chinese Musical Therapeutic Association. In this set, two different songs (Yin and Yang) are composed, which can be applied based on syndrome differentiation (Table 1). 
Table 1. FPMT used for pattern differentiation and music selection.

\begin{tabular}{|c|c|c|c|c|c|}
\hline $\begin{array}{l}\text { Music } \\
\text { Scales }\end{array}$ & Nature & Name & Meaning & Effect & Indication \\
\hline Gong & Yang & $\begin{array}{ll}\text { Shining } & \text { Sun } \\
\text { above } & \text { the } \\
\text { Dantian } & \end{array}$ & $\begin{array}{l}\text { The shining sun like a ball } \\
\text { of fire, warms and } \\
\text { eliminates dampness }\end{array}$ & $\begin{array}{l}\text { Warming the spleen } \\
\text { and lifting Yang Qi }\end{array}$ & $\begin{array}{l}\text { Poor appetite, fatigue, } \\
\text { depression, diarrhea, } \\
\text { sunken organs }\end{array}$ \\
\hline $\begin{array}{l}\text { Spleen } \\
\text { Stomach }\end{array}$ & Yin & $\begin{array}{l}\text { Jade Fluids } \\
\text { Returning to } \\
\text { Dantian }\end{array}$ & $\begin{array}{l}\text { The jade fluids like a cool } \\
\text { and sweet spring water } \\
\text { nourish stomach }\end{array}$ & $\begin{array}{l}\text { Clearing fire, } \\
\text { harmonizing stomach, } \\
\text { and removing food } \\
\text { stagnation }\end{array}$ & $\begin{array}{l}\text { Epigastric distention, } \\
\text { depressed stomach fire }\end{array}$ \\
\hline $\begin{array}{l}\text { Shang } \\
\text { Metal }\end{array}$ & Yang & $\begin{array}{l}\text { Bell and } \\
\text { Drum Music } \\
\text { in Evening } \\
\text { with Sunset } \\
\text { Glow }\end{array}$ & $\begin{array}{l}\text { The bell and drum voices } \\
\text { are spreading under the } \\
\text { setting sun kindling the sky }\end{array}$ & $\begin{array}{l}\text { Supplementing lung } \\
\text { Qi, unblocking chest } \\
\text { and consolidating } \\
\text { Wei-defensive Qi }\end{array}$ & $\begin{array}{l}\text { Wheezing, too weak to } \\
\text { spit phlegm, sweating, } \\
\text { aversion to wind }\end{array}$ \\
\hline $\begin{array}{l}\text { Large } \\
\text { Intestine }\end{array}$ & Yin & $\begin{array}{l}\text { Autumn } \\
\text { Breeze and } \\
\text { Morning } \\
\text { Dew }\end{array}$ & $\begin{array}{l}\text { In the midnight of a clear } \\
\text { autumn with full moon, it's } \\
\text { slightly cold. Dew is } \\
\text { produced to moisture the } \\
\text { leaves }\end{array}$ & $\begin{array}{l}\begin{array}{l}\text { Generating Yin and } \\
\text { clearing heat, } \\
\text { nourishing lungs and } \\
\text { generating fluids }\end{array}\end{array}$ & $\begin{array}{l}\text { Dry cough, fever and } \\
\text { restlessness }\end{array}$ \\
\hline
\end{tabular}




\begin{tabular}{|c|c|c|c|c|c|}
\hline $\begin{array}{l}\text { Jue } \\
\text { Wood }\end{array}$ & Yang & $\begin{array}{l}\text { Warm } \\
\text { Breeze under } \\
\text { Dark Sky }\end{array}$ & $\begin{array}{l}\text { Warm and comfort wind } \\
\text { breezes in spring, with } \\
\text { bright sunshine and full of } \\
\text { vigor }\end{array}$ & $\begin{array}{l}\text { Supplementing liver } \\
\text { Qi, expelling cold and } \\
\text { alleviating depression }\end{array}$ & $\begin{array}{l}\text { Dizziness, vertigo, } \\
\text { dreaminess, and } \\
\text { numbness of limbs }\end{array}$ \\
\hline Bladder & Yin & $\begin{array}{l}\text { Green } \\
\text { Leaves and } \\
\text { Wondering } \\
\text { Cloud }\end{array}$ & $\begin{array}{l}\text { Spring wind brings cool } \\
\text { and cold, green leaves }\end{array}$ & $\begin{array}{l}\text { Cooling liver fire, } \\
\text { calming hyperactive } \\
\text { liver Yang }\end{array}$ & $\begin{array}{l}\text { Vertigo, headache, } \\
\text { restlessness, red face and } \\
\text { eyes, insomnia, and } \\
\text { dreaminess }\end{array}$ \\
\hline $\begin{array}{l}\text { Fire } \\
\text { Heart }\end{array}$ & Yang & $\begin{array}{l}\text { Green and } \\
\text { Fragrant } \\
\text { Lotus under } \\
\text { Summer } \\
\text { Sunshine }\end{array}$ & $\begin{array}{l}\text { Heat in Summer, Fragrance } \\
\text { of Lotus }\end{array}$ & $\begin{array}{l}\text { Supplementing the } \\
\text { heart Yang, nourishing } \\
\text { the heart and calming } \\
\text { Shen }\end{array}$ & $\begin{array}{l}\text { Palpitation, upset, chest } \\
\text { fullness and short of } \\
\text { breath, insomnia and } \\
\text { dreaminess }\end{array}$ \\
\hline $\begin{array}{l}\text { Small } \\
\text { Intestines }\end{array}$ & Yin & $\begin{array}{l}\text { Rainbow } \\
\text { after Raining }\end{array}$ & $\begin{array}{l}\text { Vivid and clear rainbow } \\
\text { after rainbow }\end{array}$ & $\begin{array}{l}\text { Clearing heart fire, and } \\
\text { calming Shen }\end{array}$ & $\begin{array}{l}\text { Restlessness and fever, } \\
\text { red face and thirst }\end{array}$ \\
\hline $\begin{array}{l}\text { Water } \\
\text { Kidney } \\
\text { Urinary } \\
\text { Bladder }\end{array}$ & Yang & $\begin{array}{l}\text { Sunshine in } \\
\text { Winter }\end{array}$ & Warm sun at noon in winter, & $\begin{array}{l}\text { Warming and } \\
\text { supplementing kidney } \\
\text { Yang, consolidating } \\
\text { essence and } \\
\text { strengthening Qi }\end{array}$ & $\begin{array}{l}\text { Lower back pain and } \\
\text { weakness of knees, afraid } \\
\text { of cold, cold extremities, } \\
\text { spermatorrhea, } \\
\text { impotence, cold in uterus, } \\
\text { morbid leukorrhea }\end{array}$ \\
\hline
\end{tabular}


Snowing and

Yin
Frozen

Winter
Clear heart fire, Restlessness, vertigo, nourishing the kidneys tinnitus, spermatorrhea, and consolidating will amenorrhea. 


\section{Application of FPMT}

\section{Composing of Selecting Music Based on the Pattern (Zheng)}

Pattern is the diagnostic result from clinical data assessed by looking, smelling and listening, asking, and palpation. After the pattern of an illness is determined, treatment principle and specific modalities e.g. herbal remedy, acupuncture, diet, Qigong, music can be applied (Fang et al., 2013). Among the methods of analyzing pattern, Zang-fu Bian Zhen or Pattern Differentiation plays the most important role in clinical practice. Based on the relationship of the five scales and five Zang-organs, music is composed or selected according to its nature. For example, when the liver Qi is injured by an excessive anger and the symptoms, such as bitter taste in mouth, red eyes, rib-side pain, irritability, insomnia, irregular menstruation etc. are present, Jue Music should be used. The relationship of the music scales and organs was showed in figure 1. Besides, if the patient shows the same symptoms and patterns but not resulting from the emotional disorder, music therapy is also effective.

\section{Combination/Sheng and Ke}

As the music therapy and pattern differentiation of patients' symptoms are based on the theory of five phases. The condition of the pattern must be determined first ( $\mathrm{Wu}$ et al., 2012). In the diagnostics of CM, all conditions can be classified into Xu (deficiency) and Shi (excess) patterns (Grognet, 2007). According to the clinical therapeutic principle, the mother phase must be supplemented for Xu pattern while the child phase must be reduced for Shi pattern (Figure 4).
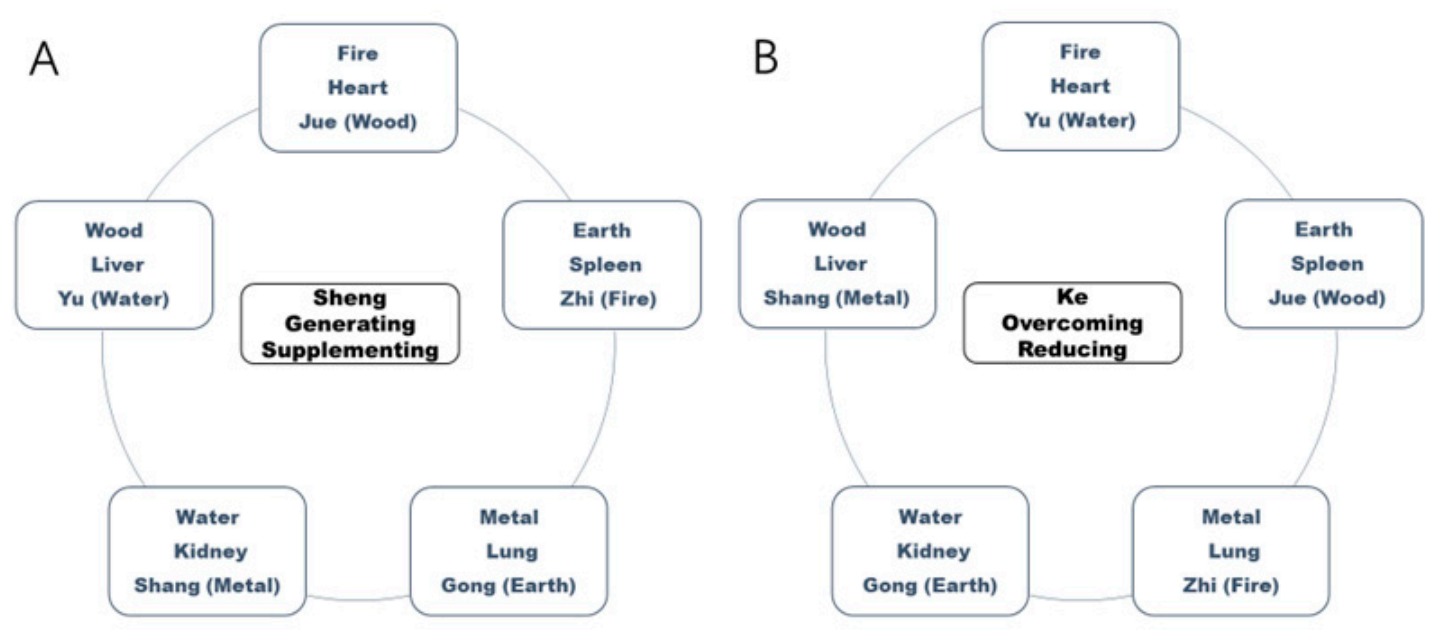
Figure 4. Music composing and selecting model based on the Sheng and Ke principle in the theory of the five phases in Chinese Medicine.

\section{Researches}

Music therapy has been reported as an effective modality in the treatment of neurological, psychological and psychiatric disorders, which are regarded as Shen disorders in CM. Music therapy is found to have positive effects on decreasing national anxiety score (Dai, 2016). There is evidence of music-supported therapy's positive effects, supporting its use for the treatment of stroke-induced motor dysfunction (Zhang 2016). (Linnemann et al.,2015), It is recorded that listening to music in daily life improves perceived control over pain in female FMS patients. There are also abundant reports on the clinical application of FPMT. The FPMT has been widely used in the treatment of depression, anxiety, PMS, chronic and pain etc., solely or in combination with other therapies(Zhang et al.,2014). Researchers have pointed out that FPMT could alleviate the anxiety and depression symptoms and improve quality of life in lung cancer patient after chemotherapy (Wang et al., 2011, Liao et al., 2011, Liao et al., 2013, Tao et al., 2016). FPMT could alleviate the symptoms of seasonal affective disorder in the elderly (Liu et al., 2014). It is usually applied in combination with acupuncture. For instance, (Li et al, 2015) holds that the combination of FPMT plus electro-acupuncture could reduce the neonatal acidosis and suffocation during normal childbirth. FPMT together with acupuncture, Tuina, Tai Chi, and Qigong have represented adjunctive benefits for the symptoms of cancer patients from the meta-review of 67 RCTs (Tao et al., 2016). FPMT is also known to improves the condition of chronic fatigue syndrome (CFS). Wu et al found that FPMT could regulate the emotion of CFS patients, relieve depression and anxiety, improve the fatigue symptoms (Wu,et al., 2009). Researchers have tried to explore the neurological mechanism of FEMP on animals. (Li and Qi, 2009) reported FPMT tended to increase the value of OE\%, as well as the crossing and rearing movement times on SD rats. (Qi et al., 2008) found that the combination of FEMP or electronic acupuncture could lower the 5-HT level in hypothalamus of anxiety rat.

\section{Conclusion}

Music therapy has served as complementary and alternative medicine for various psychological and pathological disorders. FEMP is a unique method from the combination of Chinese music and CM. It has a self-contained theory and practice. Abundant clinical trials have showed its benefits as primary or complementary approach. More systemic clinic research must be performed to validate and optimize its routines and biological and neurological mechanism must be further conducted. 
Besides, it may be a new therapy in conventional music therapy. As CM is getting more and more popular worldwide, introducing FEMP to the world may attract more attention as an effective music therapy.

\section{Acknowledgment}

This study is sponsored by Sichuan Chinese Medicine Culture Cooperation and Development Grant (ZYYWH1619) and Fund of Chengdu University of TCM (ZRQN1541).

\section{Conflict of Interests}

The author declares no conflict of interests.

\section{References}

Brandes, V., Terris, D. D., Fischer, C., Schuessler, M. N., Ottowitz, G., Titscher, G., ... Thayer, J. F. (2009). Music programs designed to remedy burnout symptoms show significant effects after five weeks. Annals of the New York Academy of Sciences, 1169(1), 422-425.

Castillo-Pérez, S., Gómez-Pérez, V., Velasco, M. C., Pérez-Campos, E., and Mayoral, M. A. (2010). Effects of music therapy on depression compared with psychotherapy. Arts in Psychotherapy, 37(5), 387-390.

Cui, M. H., Opoku Agyeman, M., Knox, D. (2016). A cross-cultural study of music in history. International Journal of Culture and History, 2(2), 65-69.

Dai, Y.Q., Chen.F., and Liu, J. (2016). Application of the five-elements music therapy in depression after myocardial infarction. China Medical Herald, 13(7), 80-89.

Fang, J., Zheng, N., Wang, Y., Cao, H., Sun, S., Dai, J., Li Q, Zhang, Y. (2013). Understanding Acupuncture Based on ZHENG Classification from System Perspective. Evidence-Based Complementary and Alternative Medicine, 2013(2), 956967.

Flower, A., Lewith, G., Liu, J. P., Gibbs, R., and Hicks, J. (2016). Applying the principles of the Five Phase (Wu Xing) model to inform good practice for studies of Chinese herbal medicine. European Journal of Integrative Medicine, 8(3), 191-197. 
Furuya, S. (2013). Music therapy as complementary and alternative medicine for neurological disorders. Neuro-Ophthalmology Japan, 30(3), 267-272.

Gong, S. (2003). Changes of the Temporal-spatial Distribution of Epidemic Disasters in 770BC-AD1911 China. Acta Geographica Sinica, 8(6), 870-878.

Gouk, P. (2015). An Enlightenment proposal for music therapy: Richard Brocklesby on music, spirit, and the passions. Progress in Brain Research, 217, 159-185.

Grognet, J. (2007). Xie's Veterinary Acupuncture. Canadian Veterinary Journal, 48(9), 934.

Hsu, E. (2000). Spirit (shen), styles of knowing, and authority in contemporary Chinese Medicine. Culture, Medicine, and Psychiatry, 24(2), 197-229.

Karchmer, E. I. (2013). The excitations and suppressions of the times: locating the emotions in the liver in modern Chinese medicine. Culture, Medicine, and Psychiatry, 37(1), 8.

Kim, J.(2013). Music Therapy. Encyclopedia of Autism Spectrum Disorders, 9(2), 1961-1966.

Koelsch, S. (2014). Brain correlates of music-evoked emotions. Nature Reviews Neuroscience, 15(3), 170.

Li, L., and Qi, J. (2009). Researching on five elements music to influence the behavior of anxiety disercler rats. Journal of Sichuan of Traditional Chinese Medicine, 27(7), 24-26.

Li, L., Wang, M.L., Li, M.Y., Wei, J., Gan, H.S., Jiang, L.Y., He, L.Q., and Chen, L.F. (2015). The effects of five elements of music and electroacupuncture in labor analgesia on newborn blood gas analysis. Journal of Youjiang Medical University for Nationalities, 37(3), 355-356,370.

Li, X., Ma, S., CUi, J., LI, Q., and Yang, X. (2010). Enlightment for acupuncture from the Yin Yang 25 Individules in Huangdineijing. Lishizhen Medicine and Materia Medica Research, 21(4), 965-966.

Liao, J, Yang,Y.F., Zhao. Y.C., Xiao. CY, and Yu, Y. (2011). Kappa analysis on the application of selecting traditional chinese medicine five-element music under syndrome differentiation. World Science \& Technology, 13(6), 944-947. 
Liao, J., Yang, Y.H., Lorenzo C., Zhao, Y.C., Xu, Y. (2013). Effects of chinese medicine five-element music on the quality of life for advanced cancer patients: a randomized controlled trial. Chinese Journal of Integrative Medicine, 19(10), 736-740.

Linnemann, A., Kappert, M. B., Fischer, S., Doerr, J. M., Strahler, J., and Nater, U. M. (2015). The effects of music listening on pain and stress in the daily life of patients with fibromyalgia syndrome. Frontiers in Human Neuroscience, 9(July), 434.

Liu, X., Niu, X., Feng, Q., and Liu, Y. (2014). Effects of five-element music therapy on elderly people with seasonal affective disorder in a Chinese nursing home. Journal of Traditional Chinese Medicine, 34(2), 159-161.

Ma, S. L., Tang, Q. L., Liu, H. W., He, J., and Gao, S. H. (2013). Correlation analysis for the attack of bacillary dysentery and meteorological factors based on the Chinese medicine theory of Yunqi and the medical-meteorological forecast model. Chinese Journal of Integrative Medicine, 19(3), 182-186.

Maratos, A., and Gold, C. (2008). Music therapy for depression. Cochrane Database of Systematic Reviews, 108(1), CD004517.

Miller, S. M. (2013). Reflections on the musical mind: an evolutionary perspective. Computer Music Journal, 26(3), 98-100.

Moss, C. A. (1999). Five element acupuncture: treating body, mind, and spirit. Alternative Therapies in Health \& Medicine, 5(5), 52.

Qi, J., Peng, Q., Liu, J., Yang, Z., and LI, L. (2008). The effect of electronic acupuncture and five phases music therapy on the serum and hypothalamus 5-HT level on anxiety rat. Shaanxi Journal of Traditional Chinese Medicine, 29(5), $627-629$.

Scheid, V. (2013). Depression, constraint, and the liver: (dis)assembling the treatment of emotion-related disorders in Chinese medicine. Culture, Medicine, and Psychiatry, 37(1), 30-58.

Sung, K. T. (2001). Elder respect: exploration of ideals and forms in East Asia. Journal of Aging Studies, 15(1), 13-26.

Tao, W. W., Jiang, H., Tao, X. M., Jiang, P., Sha, L. Y., and Sun, X. C. (2016). Effects of acupuncture, tuina, tai chi, Qigong, and traditional Chinese medicine 
five element music therapy on symptom management and quality of life for cancer patients: a meta-analysis. Journal of Pain \& Symptom Management.

Thompson WF, and Schlaug, G. (2015). The healing power of music. Scientific American Mind, 26(2), 32-41.

Wang, M., Chen, F., \& Jiang, M. (2011). Study on traditional Chinese medical music on quality of life of anxiety-depression type of pulmonary cancer patients during chemotherapy. Chin Nurs Res, 25(9c), 2478-2479.

Wang, Q., and Zhu, Y. (2009). Epidemiological investigation of constitutional types of Chinese medicine in general population: based on 21,948 epidemiological investigation data of nine provinces in China. China Journal of Traditional Chinese Medicine \& Pharmacy, 24(1), 7-12.

Wu, L.L., Zhang, X.G., Chen, M., and Zhang, Y. (2009). Investigation of traditional chinese medicine five element music therapy in treating 60 cases of chronic fatigue syndrome emotional disorder. Guide of China Medicine, 7(24), 8-10.

Wu, T., Yang, M., Wei, H. F., He, S. H., Wang, S. C., and Ji, G. (2012). Application of metabolomics in traditional Chinese Medicine differentiation of deficiency and excess syndromes in patients with diabetes mellitus. Evidence-Based Complementary and Alternative Medicine, 2012: 968083.

Xu, B., Sui, Y., Zhu, C., Yang, X., Zhou, J., Li, L., Ren, L., Wang, X. (2017). Music intervention on cognitive dysfunction in healthy older adults: a systematic review and meta-analysis. Neurological Sciences, 1-10.

Zhang, H., Zhang, X.G., Liang, X.L., Lai, H., Gao, J., Liu, Q., Wang, H.Y. (2015). Introduction on the emotion-will overcoming therapy (ewot): a novel alternative approach of psychological treatment from chinese medicine. Chinese Medicine, 6(2), 75-82.

Zhang, H., Zhang, X.G, Wang, H.Y., Cao, B., Liu, Y., Liao, M.Y., Wang, M.Q (2014). Application of the five-element musicotherapy to emotion nursing with traditional Chinese medicine. Chinese Journal of Convalescent Medicine, 23(12), 1077-1079.

Zhang, Y., Cai, J., Zhang, Y., Ren, T., Zhao, M., and Zhao, Q. (2016). Improvement in stroke-induced motor dysfunction by music-supported therapy: a systematic review and meta-analysis. Scientific Reports, 6, 38521. 
Zhang, H., Tan, C., Wang, H., Xue, S., and Wang, M. (2010). Study on the history of traditional Chinese medicine to treat diabetes. European Journal of Integrative Medicine, 2(1), 41-46. 